Journal of Applied Fluid Mechanics, Vol. 6, No. 1, pp. 1-6, 2013.

Available online at www.jafmonline.net, ISSN 1735-3572, EISSN 1735-3645.

DOI: $10.36884 / \mathrm{jafm} .6 .01 .19474$

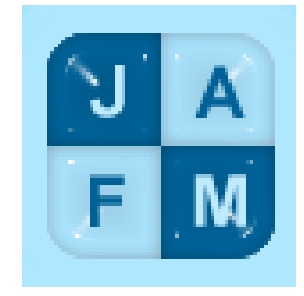

\title{
Wave Generated by the NACA4412 Hydrofoil near Free Surface
}

\author{
H. Ghassemi ${ }^{1 \dagger}$ and A. R. Kohansal ${ }^{2}$ \\ ${ }^{1}$ Department of Ocean Engineering, Amirkabir University of Technology (AUT), Tehran, Iran \\ ${ }^{2}$ Department of Marine Engineering, Persian Gulf University (PGU), Bushehr, Iran \\ †Corresponding AuthorEmail: Gasemi@aut.ac.ir
}

(Received June 10, 2009; accepted October 28, 2011)

\begin{abstract}
The generation of wave due to moving hydrofoil in steady streams close to a free surface is presented. The potential-based boundary element method is employed to the NACA4412 hydrofoil with linearized dynamic and kinematic boundary conditions on the free surface. The perturbation velocity potential is calculated using the Green formulation and Kutta condition. The numerical results of waves generated by the hydrofoil are presented and discussed at various Froude numbers and immersion depths.
\end{abstract}

Keywords: Free surface, NACA4412 hydrofoil, Boundary element method

NOMENCLATURE

$\begin{array}{ll}A R & \text { aspect ratio } \\ C & \text { chord length } \\ P & \text { pressure } \\ L & \text { lift } \\ F_{n} & \text { Froude number } \\ g & \text { gravitational acceleration } \\ G & \text { Green's function } \\ \vec{V}_{0} & \text { inflow velocity } \\ \vec{X} & \text { position vector } \\ K_{0} & \text { wave number } \\ \vec{n} & \text { outward unit normal vector } \\ h / C & \text { depth-chord ratio } \\ N_{B W} & \text { number of elements on the body } \\ N_{F W} & \text { number of elements on the free surface } \\ N_{W} & \text { number of elements on the trailing vortex } \\ & \text { wake surface }\end{array}$

\section{INTRODUCTION}

The hydrofoil is a lifting body that generates the lift to raise the craft's hull in order to decrease the wetted surface and as a result to diminish drag. The shape of hydrofoils is very important in their application in marine fields. Also, determination of the hydrofoils' hydrodynamic characteristics like forces and moments experienced by a hydrofoil of finite aspect ratio and

$\begin{array}{cl}M & \text { number and span wise of the hydrofoil } \\ N_{B} & \text { total number of the elements on the body } \\ N_{F} & \begin{array}{l}\text { total number of the elements on the free } \\ \text { surface }\end{array} \\ N_{T} & \text { total number of element } \\ \zeta & \text { wave elevation } \\ \phi & \text { perturbation potential } \\ \Phi & \text { total velocity potential } \\ \rho & \text { density of the water } \\ \alpha & \text { attack angle } \\ S_{B} & \text { surface of the body } \\ S_{F} & \text { surface of the free surface } \\ S_{W} & \text { surface of the TVW } \\ T E & \text { trailing edge }\end{array}$

arbitrary form in the proximity of a free surface and their resultant wave patterns is essential in their application. Then, the designer should choose how the hydrofoil will be arranged, what shape it will have, what geometrical angle of attack it should have, etc. Existence of the free surface will complicate the analysis. As a result, when a vortex interacts with a free surface, the velocity field is significantly altered and this results in a complex structure and dynamics.

This problem has been considered by many researchers. Yeung et al. (1979) dealt with thick hydrofoil methods 
which provided a precise representation of the flow near the hydrofoil surface. Janson (1997) applied linear and nonlinear potential flow calculations of free surface waves including lift and induced drag of hydrofoils, vertical struts and Wigley ship hulls. There have been some experimental as well as theoretical studies on the influence of different hydrofoil configurations on the hydrodynamic characteristics. Hydrodynamic analysis of two and three dimensional hydrofoils moving beneath the free surface was developed in Bal (2008); Kouh (2002); Xie et al. (2007). Bai et al. (1994) used a localized finite-element method for the nonlinear steady waves due to two-dimensional hydrofoils. Numerical calculations of ship induced waves using the boundary element method with triangular mesh surface calculated in 2000. Dawson (1977) employed a distribution of Rankine type sources on the ship hulls and free surfaces. Isparametric boundary element method is employed to the underwater body and calculated the lift, drag and wave pattern by Ghassemi et al. (2010).

The aim of this work is to investigate the wave generated by the hydrofoils moving with constant speed placed in a steady stream close to the free surface. Computational results of free surface pattern generated by NACA4412 hydrofoil are presented.

\section{Problem Definition}

Let us consider a hydrofoil that is moving at a constant forward speed in infinite deep water near the free surface, as depicted in Fig. 1. A Cartesian coordinate system $O-X Y Z$ is defined to be fixed in the space and a moving coordinate system $o-x y z$ fixed on the hydrofoil. The horizontal and vertical axes, $o x$ and $o z$, are along and at the right angle to the direction of the motion. The z-coordinate is defined as being vertically upwards from the undisturbed free surface. The fluid is assumed to be in viscid, incompressible, irrotational and without surface tension. These assumptions lead to a boundary value problem for the velocity potential with the Laplace equation satisfied in the fluid Domain. Under the global coordinate system, a total velocity potential $\Phi$ can be defined as the sum of the free-stream and the perturbation potentials as follows:

$\Phi=\phi+\vec{V}_{0} \cdot \vec{X}$

where $\phi$ is the perturbation velocity potential which is disturbance caused by the presence of a hydrofoil and is superimposed on the uniform flow and $\vec{X}$ is the position vector.

The perturbation and total potentials are governed by Laplace's equation:

$$
\nabla^{2} \Phi=\nabla^{2} \phi=0
$$

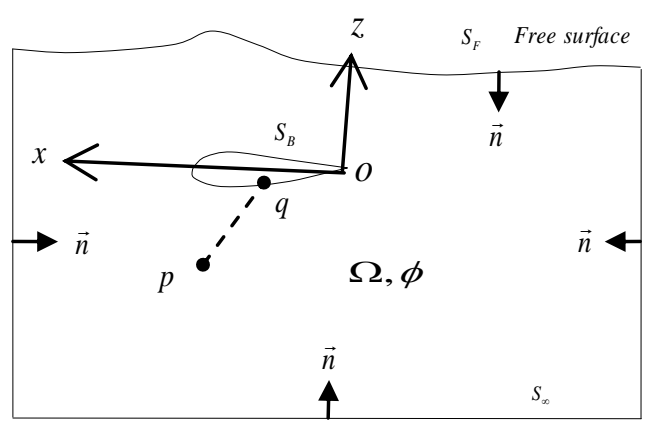

Fig. 1. Definition of coordinate system and some parameters

The potential $\phi$ is computed by the boundary element method, which is based on Green's identity. In general, the boundary surface includes the body surface $\left(S_{B}\right)$, wake surface $\left(S_{W}\right)$ and the free surface $\left(S_{F}\right)$. According to Green's third identity, the perturbation potential $\phi$ is given by the following integral expression with $q$ on surface $S$ and $p$ in $\Omega$ :

$4 \pi E \phi(p)=\int_{S}\left[\frac{\partial \phi(q)}{\partial n} G-\phi(q) \frac{\partial G}{\partial n}\right] d S$

where $S=S_{B}+S_{W}+S_{F}$ are the boundaries of the lifting body, wake and the free surface, respectively. $p$ is the field point and $E$ is the solid angle which depends on its position in the fluid domain $\Omega$. If point $p$ is placed on the boundary (body surface), then the coefficient $E$ is replaced by $1 / 2$. For $p$ inside and outside $\Omega$, its values are one and zero, respectively. $G$ is Green's function including the image body relative to the free surface. Then, the boundary conditions have to be determined. In order to satisfy also the linearized free surface boundary condition that is applicable here, it is necessary to add, for any one source strength, the influences of its image in the undisturbed free surface. In flow problems involving free surfaces, part of the boundary of the computational domain (corresponding to the free surface) is unknown and must be determined as part of the solution. On the free surface, two boundary conditions should be satisfied, the kinematic free surface boundary condition (KFSBC) and the dynamic free surface boundary condition (DFSBC).

$\left(\nabla \phi-\vec{V}_{0}\right) \cdot \nabla \varsigma=\phi_{z} \quad$ on $\quad z=\varsigma(x, y)$

where $\zeta$ denotes the wave elevation and can be expressed as

$\varsigma=\frac{1}{g}\left(-\vec{V}_{0} \cdot \nabla \phi+\frac{1}{2} \nabla \phi \cdot \nabla \phi\right) \quad$ on $\quad z=\varsigma(x, y)$ 
where $g$ is the gravitational acceleration. Fig. 2 shows the boundary conditions on the body and free surface.

The free surface formulation (Eq. (4)) is nonlinear. Here, the linearized boundary-value problem is used by omitting the nonlinear terms in the boundary conditions. Then, the linearized boundary conditions are satisfied on the undisturbed free surface

$$
\begin{aligned}
& -V_{0} \cdot \nabla \zeta=\phi_{z} \quad \text { on } \quad z=0, \\
& \zeta=-\frac{1}{g} V_{0} \phi_{x} \quad \text { on } \quad z=0,
\end{aligned}
$$
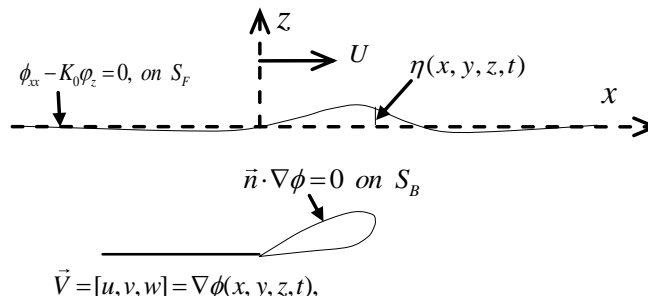

\section{$\nabla^{2} \phi(x, y, z, t)=0$,}

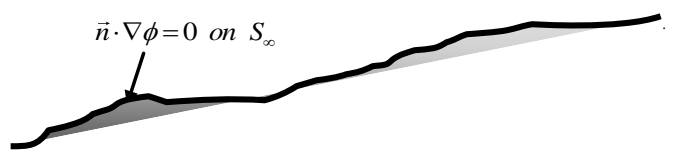

Fig. 2. The governing equation and boundary conditions

Inserting Eq. (6) into Eq. (7), a composed boundary condition on the free surface is obtained as:

$V_{0}^{2} \phi_{x x}=g \phi_{z} \quad$ on $\quad z=0$,

In this study to compute the free surface boundary condition (Eq. 8), a four-point upwind difference scheme is used to compute $\phi_{x x}$. The flow velocity normal to the surface is zero.

$\frac{\partial \phi}{\partial n}=-\vec{V}_{0} \cdot \hat{n}$

$\hat{n}=\left(n_{x}, n_{y}, n_{z}\right)$ is the outward unit normal vector, defined positive when pointing into the fluid region. Also at infinity: $\lim |\nabla \phi|=0, \quad$ when $r \rightarrow \infty$,

And the separation of the flow corresponds to the flow at the trailing edge on the classical airfoil theory and is secured through the Kutta condition. According to this condition, the velocity in the trailing edge is the same in the upper and lower surfaces. Once the system of equations which is constructed based on the boundary conditions has been solved, the potential, velocity and pressure on the hydrofoil can be computed.

\section{Numerical Method}

The body surface and free surface are discretized into the quadrilateral elements. A typical meshing on a body surface and the number of elements are shown in Figs. 3 and 4 respectively.

It is assumed that the hydrofoil is subdivided into $M$ spanwise strips which are extended from tip to tip and $N$ chordwise strips which are extended from the leading edge to the trailing edge, giving a total of $M \times N$ elements. The discretized form of integral Eq. (3) for the wetted surface of the body and free surface are expressed and integrals involved in the above equation are numerically calculated by the Gauss quadrature integration method.

A numerical code incorporating a low-order boundary element formulation has been developed. The method is potential based employing both doublets and sources distributed on the foil and cavity surfaces. The source strengths are unknown and should be calculated during computation of integrals.

The discretization of the wetted body surface and free surface involves consideration of several issues. The Numerical differentiation of the surface potential is required to calculate surface velocity.

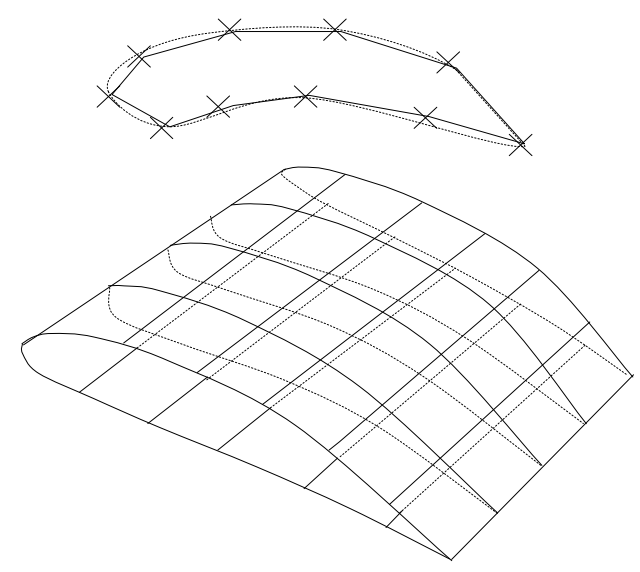

Fig.3. Modeling of hydrofoil surface by quadrilateral elements 


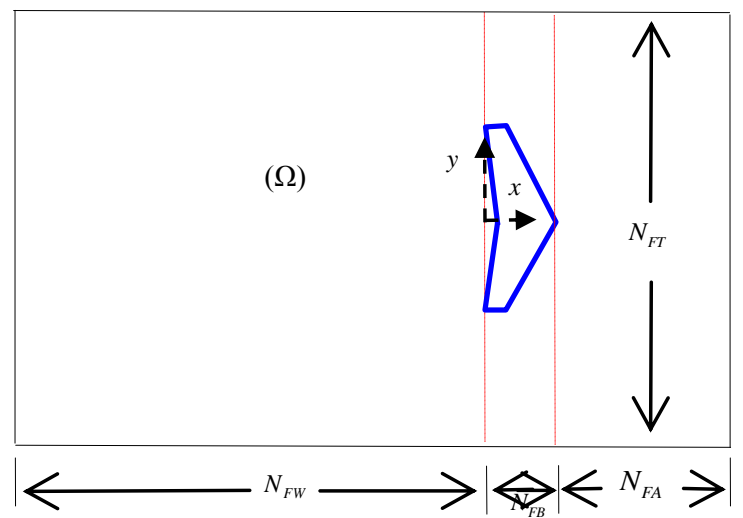

Fig. 4. The number of elements on the free surface

\section{Results AND Discussions}

A rectangular type hydrofoil shaped with NACA4412 sections is selected. The hydrofoil moves at a constant speed beneath the free surface. The effect of the submerged depth $(h / C)$, aspect ratio (A.R.) and Froude number $\left(F_{n}\right)$ on the wave pattern are calculated. The Froude number is based on the mean chord length. Results are presented for Froude numbers from 0.5 to 1. A three dimensional hydrofoil with NACA4412 has a nonsymmetric profile and we focused on this to compute more results with the present method. Figure 5 shows the surface mesh of the NACA4412 foil and aspect $\operatorname{ratio}(A R=4)$.

This numerical instability is due to the spacing of the foil and upper cavity surfaces relative to the length of the elements in the vicinity. For the present analysis, the foil wetted surface and free surface have been discretized with 900 and 2700 quadrilateral elements, respectively. The greater number of elements means a more stable solution. In Fig. 6 , for this foil at depth ratio $(h / C=1)$, attack angle ( $\alpha=5[\mathrm{deg}]$.$) and Froude number \left(F_{n}=1\right)$, the computational results of the center plane wave profile are compared with calculated values given by Kouh et. al. (2002) and Xie et al. (2007). The present calculation results are well correlated with the experimental data.

The waves generated of the hydrofoils are calculated by the present method in various conditions. The perspective view of the free surface waves are given in various conditions. In Figs. 7 to 12 the calculated wave generated for rectangular hydrofoils with several Froude numbers and depth of submergence are shown. It can be seen that

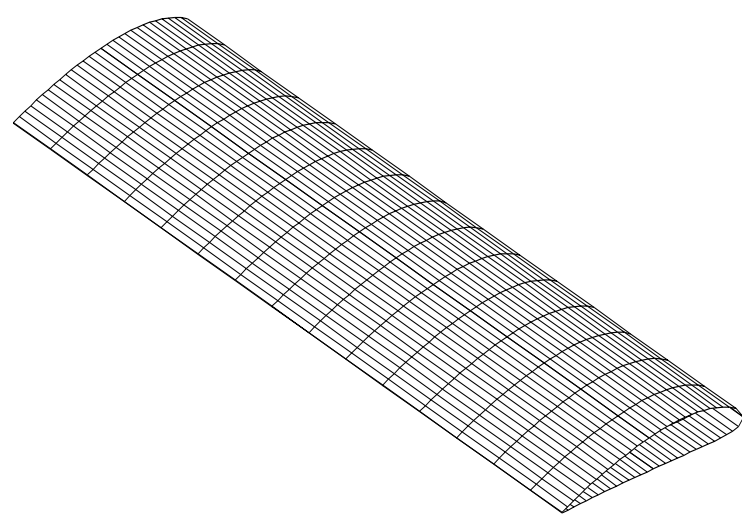

Fig. 5. Mesh surface of the rectangular hydrofoil

the effect of immersion on the hydrodynamic performance is significant when the hydrofoil is located near the free surface. Figure 12 shows the wave generated by two parallel hydrofoils. It is observed that the wave pattern interacted by two source pressure due to the hydrofoil.

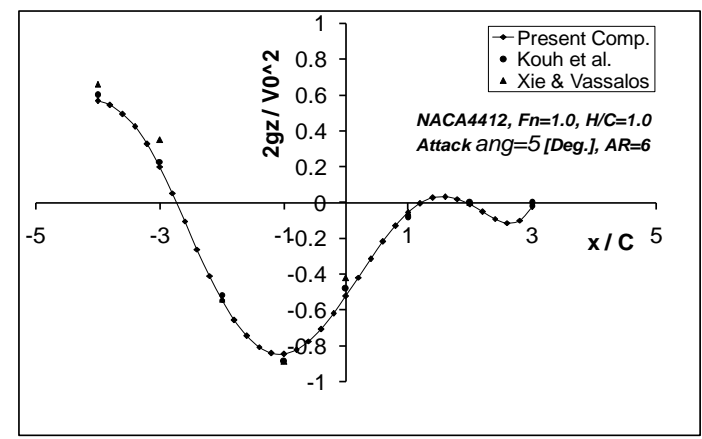

Fig. 6. Comparison of wave profiles at the center plane of the hydrofoil

For the second choice, a delta type hydrofoil is selected. Figure 13 shows the surface mesh of the NACA4412 delta shape hydrofoil. Calculations are made for this hydrofoil in different conditions. Figures 14 and 15 illustrate the variation of the wave patterns with respect to the Froude number at two depth ratios on the rectangular and delta hydrofoils with a NACA4412 profile. These figures give a fair impression about the wave pattern close to the body surface. 
H. Ghassemi et al. / JAFM, Vol. 6, No. 1, pp. 1-6 2013.

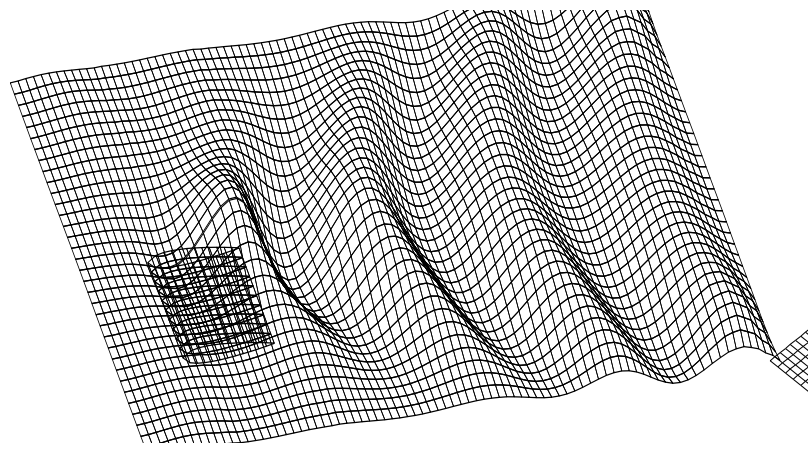

Fig. 7. Wave generated by the rectangular hydrofoil

$$
h / C=0.8, F_{n}=0.51
$$

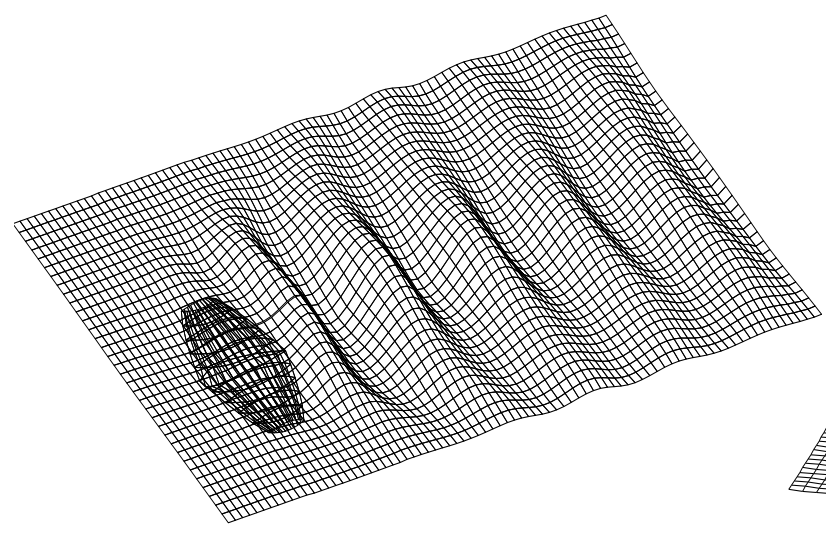

Fig. 8. Wave generated by the non-rectangular hydrofoil $h / C=0.8, F_{n}=0.574$

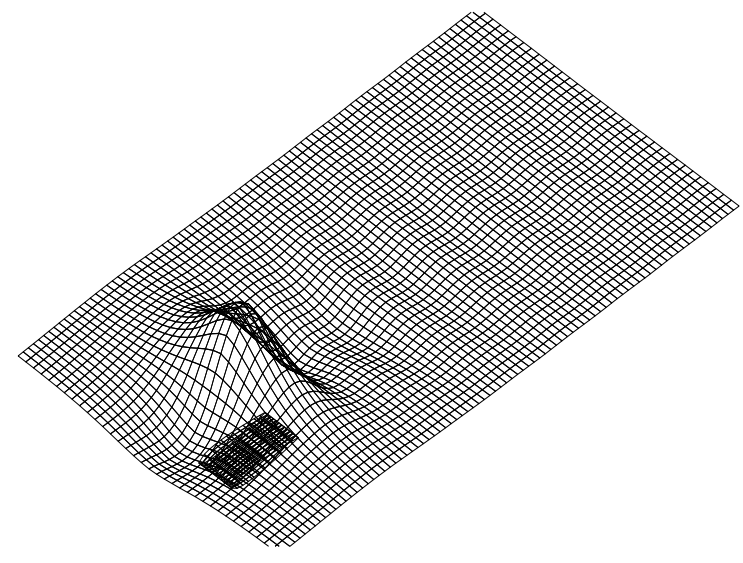

Fig. 9. Wave generated by the rectangular hydrofoil, $h / C=0.7, F_{n}=0.606$

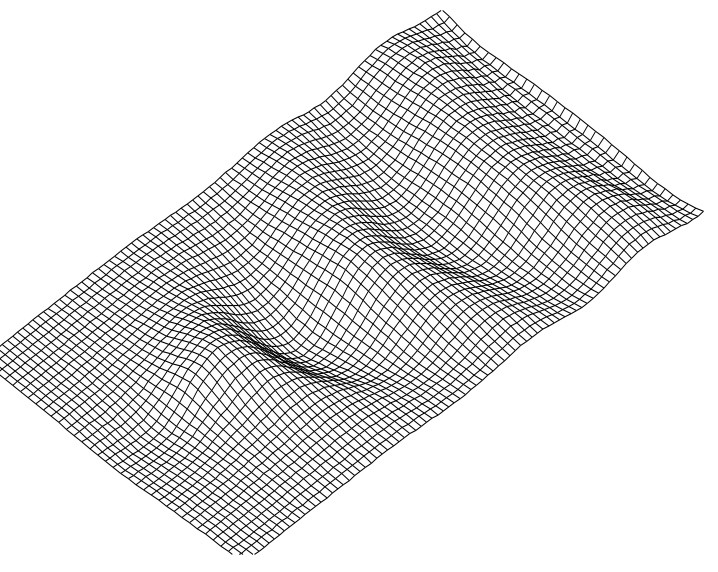

Fig. 10. Wave generated by the rectangular hydrofoil, $h / C=0.7, F_{n}=0.383$

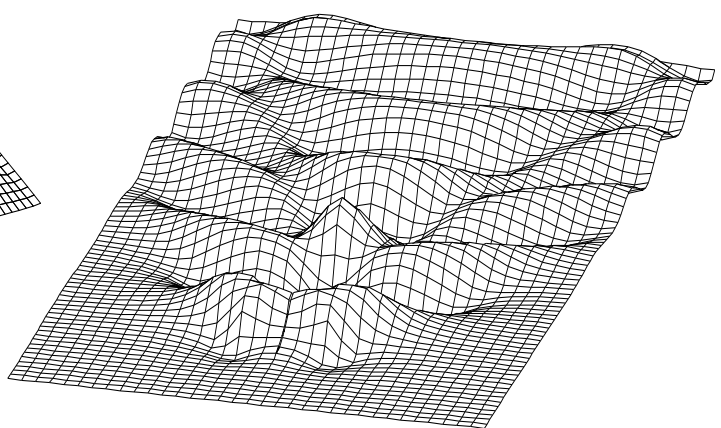

Fig. 11. Wave generated by two parallel hydrofoil, $h / C=0.5, F_{n}=0.7$

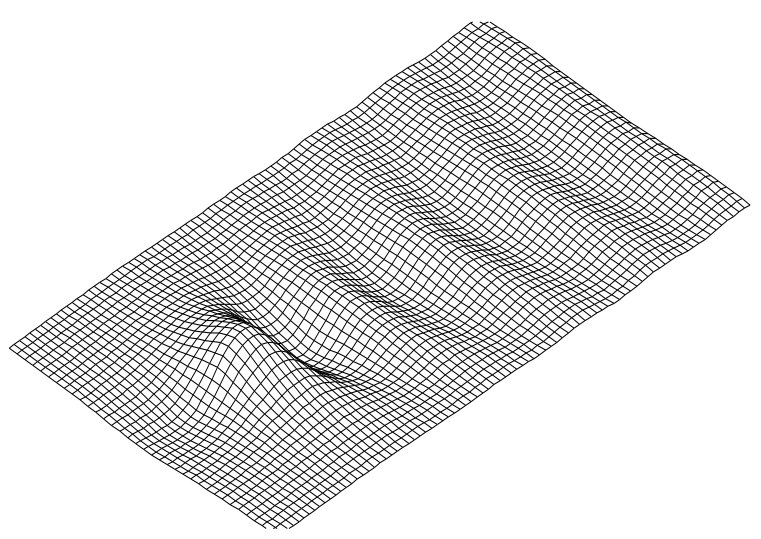

Fig. 12. Wave generated for the rectangular hydrofoil, $h / C=0.7, F_{n}=0.447$ 


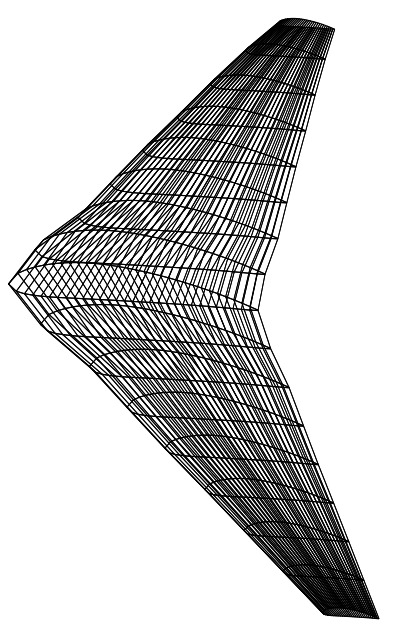

Fig. 13. Mesh surface of the delta hydrofoil

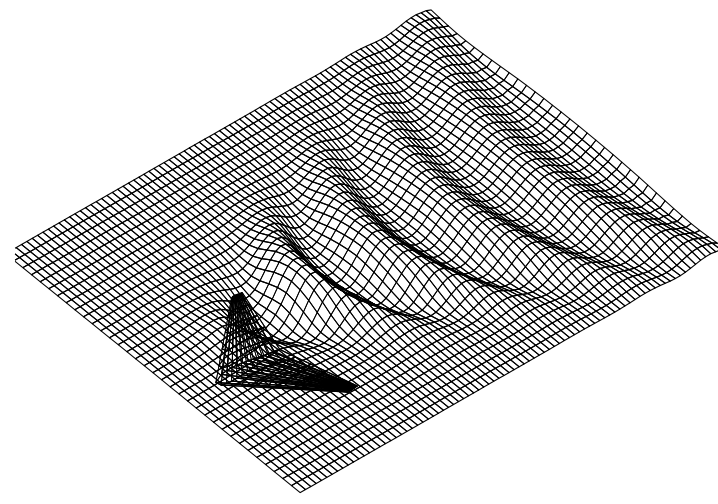

Fig. 14. Wave generated by the delta hydrofoil, $h / C=0.5, \alpha=5[\mathrm{deg}],. F_{n}=0.4$

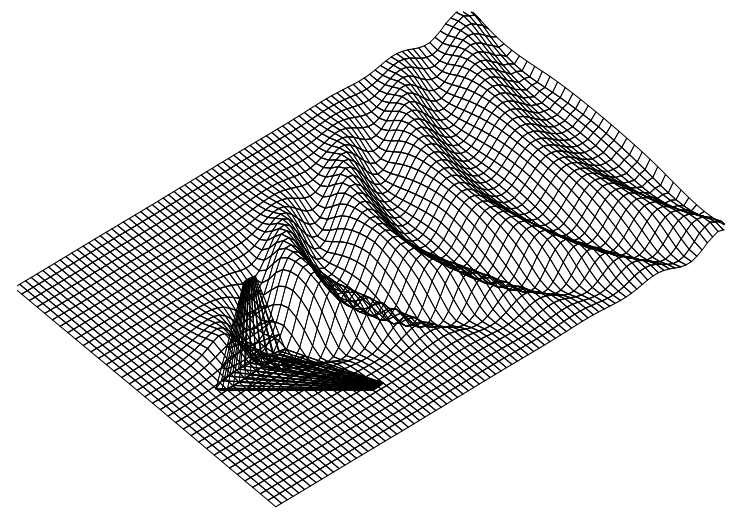

Fig. 15. Wave generated by the delta hydrofoil, $h / C=0.5, \alpha=5[\mathrm{deg}],. F_{n}=0.7$

\section{CONCLUSION}

We have calculated the wave generated by the hydrofoils moving at a constant speed near the free surface. A number of numerical tests were carried out for rectangular and delta shape hydrofoils with NACA4412 section in different operational conditions. By comparing the results of wave elevation with those of experiments and other numerical values, it is revealed that the method can simulate the wave patterns well.

\section{ACKNOWLEDGMENTS}

This work was supported by AUT and the Marine Research Center Foundation.

\section{REFERENCES}

Bai, K.J. and Han, J.H. (1994). A localized finite-element method for the nonlinear steady waves due to a twodimensional hydrofoil. J. Ship Res. 38, 42-51.

Bal, S. (2008). Prediction of wave pattern and wave resistance of surface piercing bodies by a boundary element method. Int. J. Numer. Meth. Fluids, 56(3), 305-329.

Dawson, C. W. (1977). A practical computer method for solving ship-wave problems. Proceedings of Second International Conference on Numerical Ship Hydrodynamics, 30-38.

Ghassemi, H. and Kohansal, M.R. (2010) Isoparametric boundary element method applied to the rectangular and delta hydrofoils near free surface, China Ocean eng., 24(2)

Janson, C.E. (1997). Potential flow panel method for the calculation of free surface flows with lift, $\mathrm{Ph}$. D. thesis, Chalmers University of Technology.

Kouh J. S. (2002). Performance analysis of two dimensional hydrofoil under free surface. Journal of National Taiwan University 86.

Xie, N. and Vassalos, D. (2007). Performance analysis of 3D hydrofoil under free surface. Ocean Engineering, 34, 1257-1264.

Yeung, R. W. and Bouger, Y. C. (1979). A hybrid-integral equation method for steady two-dimensional ship waves. Int. J. Num. Meth. Eng., 14, 317-336. 